\title{
Crowned Dens Syndrome as an Initial Manifestation of Crystalline Deposition Disease
}

\author{
Ryo Koda ${ }^{1,2}$, Yohei Tsuchida ${ }^{1}$, Kazutaka Yoshizawa ${ }^{1}$, Kazuo Suzuki ${ }^{1}$, Akio Kasai ${ }^{1}$, \\ Tetsuro Takeda ${ }^{2}$, Junichiro James Kazama ${ }^{3}$, Ichiei Narita ${ }^{3}$ and Kazukiyo Yoshida ${ }^{1}$
}

\begin{abstract}
An 88-year-old woman presented with fever and acute posterior neck pain. A CT scan revealed calcification of the transverse ligament and crown-like calcification around the odontoid process. According to the clinical and radiological findings, she was diagnosed with crowned dens syndrome (CDS). Her symptoms drastically improved following treatment with oral nonsteroidal anti-inflammatory medication. An X-ray of her wrist, elbow, shoulder and knee joints showed asymptomatic calcium deposits, suggesting underlying crystalline deposition disease. CDS may occur as the initial presentation of crystalline deposition disease. The measurement of procalcitonin and an X-ray survey of the major joints may be helpful for the diagnosis of CDS.
\end{abstract}

Key words: crowned dens syndrome, crystalline deposition disease, nonsteroidal anti-inflammatory drugs

(Intern Med 54: 2405-2408, 2015)

(DOI: 10.2169/internalmedicine.54.4571)

\section{Introduction}

Crowned dens syndrome (CDS) is a relatively rare clinical condition that is characterized by the unique radiographic feature of a crown-like calcium deposition around the odontoid process (1). Symptoms of CDS include high fever, acute posterior neck pain, or sometimes neck rigidity and Kernig's sign (2). These manifestations are quite misleading since high fever with neck pain is typically suggestive of infectious meningitis (2). However, considering CDS in the differential diagnosis of feverish acute neck pain is important because the diagnostic and therapeutic strategies vary significantly between meningitis and CDS. Unnecessary invasive diagnostic procedures, or indiscreet antibacterial or anti-viral treatment, are ineffective and may be potentially harmful for patients with CDS.

We herein describe the case of an 88-year-old Japanese woman who presented with a sudden onset of posterior neck pain and high fever. Infectious disease was initially suspected, however, the administration of intravenous antibiotic agents was ineffective. Furthermore, the patient's serum pro- calcitonin level was not elevated, thus suggesting an infectious etiology to be less likely. A CT scan of the neck revealed linear calcification in the transverse ligament of the atlas and crown-shaped calcifications around the odontoid process. Although asymptomatic, an X-ray revealed significant calcium deposits in both sides of wrist, elbow, shoulder and knee joint, suggesting the presence of underlying crystalline deposition disease. A diagnosis of CDS was made and nonsteroidal anti-inflammatory drugs (NSAIDs) treatment remarkably alleviated the patient's symptoms and inflammatory reaction.

\section{Case Report}

An 88-year-old Japanese woman with a history of hypertension and duodenal ulcer visited our hospital due to acute onset of fever and sharp pain in the posterior neck. The onset of neck pain was one day before visitation. The patient had no prior history of traumatic injury and no apparent history of pseudogout attack. The patient's family history was unremarkable. On the first visit, her consciousness was clear. Her body temperature was $38.3^{\circ} \mathrm{C}$, blood pressure

${ }^{1}$ Niigata Prefectural Muikamachi Hospital, Japan, ${ }^{2}$ Dokkyo Medical University Koshigaya Hospital, Japan and ${ }^{3}$ Niigata University Graduate School of Medical and Dental Sciences, Japan

Received for publication November 23, 2014; Accepted for publication January 13, 2015

Correspondence to Dr. Ryo Koda, ryokouda@gmail.com 

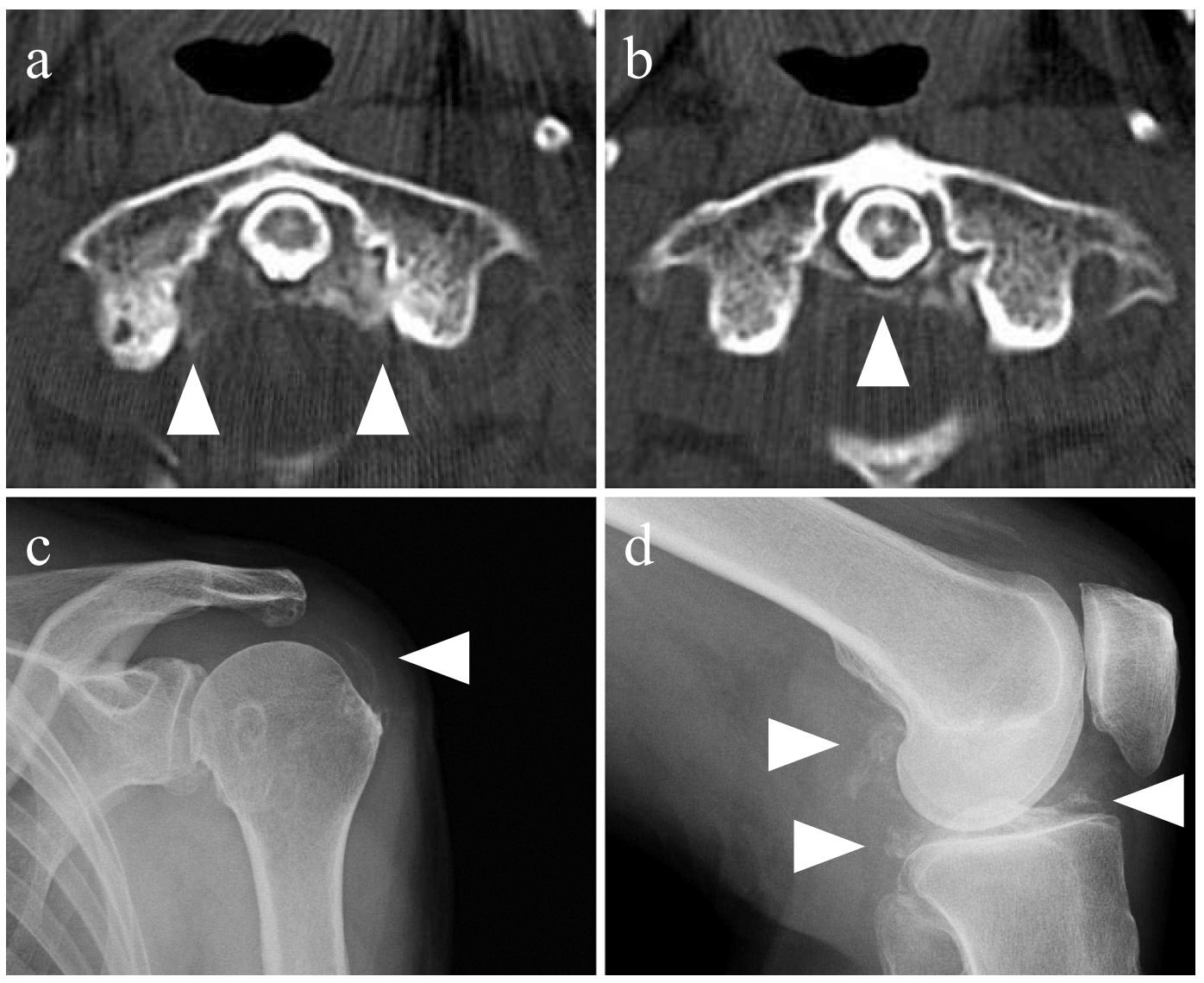

Figure 1. A cervical CT scan revealed crown-like calcium deposits around the odontoid process (1a, arrowheads). Calcification of the transverse ligament of the atlas was prominent (1b, arrowhead). Although the patient was asymptomatic, calcium deposits were apparent in the left shoulder (1c, arrowhead) and left knee joint (1d, arrowheads).

$160 / 95 \mathrm{mmHg}$, and pulse 103 beats per minute with a regular rhythm. On the physical examination, auscultation of the chest and lungs was normal and abdominal tenderness, masses, or vascular bruit was not apparent. The superficial lymph nodes were not palpable. Furthermore, edema formation or skin eruption was not noted in her extremities and palpation of the temporal artery was normal. Neck rigidity or Kernig's sign was not observed. The peripheral joints were not tender or deformed. The patient did not have any neurological deficit; however, she felt sharp pain in her posterior neck with movement. Due to this annoying pain, her range of neck motion was restricted. The next day, she was admitted to our hospital for further work-up. The major laboratory findings were as follows: white blood cell count (WBC) $11,300 / \mu \mathrm{L}$, hemoglobin $11.2 \mathrm{~g} / \mathrm{dL}$, platelets $41.7 \times$ $10^{4} / \mu \mathrm{L}$, aspartate aminotransferase (AST) $23 \mathrm{IU} / \mathrm{L}$, alanine transaminase (ALT) $18 \mathrm{IU} / \mathrm{L}$, lactate dehydrogenase (LDH) $202 \mathrm{IU} / \mathrm{L}$, alkaline phosphatase (ALP) $147 \mathrm{IU} / \mathrm{L}$, blood urea nitrogen (BUN) $18.1 \mathrm{mg} / \mathrm{dL}$, creatinine $0.58 \mathrm{mg} / \mathrm{dL}$, total protein $6.7 \mathrm{~g} / \mathrm{dL}$, albumin $1.85 \mathrm{~g} / \mathrm{dL}$, uric acid $3.9 \mathrm{mg} / \mathrm{dL}$, ferritin $96.0 \mathrm{ng} / \mathrm{mL}$, C-reactive protein (CRP) $9.62 \mathrm{mg} / \mathrm{dL}$ (normal range: $<0.40$ ), erythrocyte sedimentation rate (ESR) $106 \mathrm{~mm} / \mathrm{hr}$, immunoglobulin G (IgG) $807 \mathrm{mg} / \mathrm{dL}, \mathrm{C} 3192.7$ $\mathrm{mg} / \mathrm{dL}$ (normal range: 75.0-148.0), and C4 $36.8 \mathrm{mg} / \mathrm{dL}$ (normal range: 14.0-38.0). The serology for hepatitis $\mathrm{B}$, hepatitis
C, anti-nuclear antibody (ANA), anti-double stranded DNA antibody, myeloperoxidase and proteinase 3 anti-neutrophil cytoplasmic antibody (ANCA) was negative. The serum level of rheumatoid factor was slightly increased (19.0 IU/ $\mathrm{mL}$; normal range: $<15.0$ ); however, anti-cyclic citrullinated peptide (CCP) antibody was negative $(0.8 \mathrm{U} / \mathrm{mL}$; normal range: $<4.5)$. The urinalysis findings were normal. These laboratory findings were not suggestive of any underlying connective tissue disease or vasculitis.

Initially, a bacterial infection was suspected and the patient was treated with antibiotics (ceftriaxone sodium hydrate, $2.0 \mathrm{~g}$ per day intravenously and meropenem hydrate, $1.0 \mathrm{~g}$ per day intravenously). However, the antibiotic treatment was not effective for her fever and neck pain. A blood culture was negative. An enhanced CT scan of the chest, abdomen, and pelvis revealed no possible focus of infection or malignancy. Furthermore, an echocardiogram showed no apparent mitral or aortic valve vegetation. Additionally, the serum procalcitonin level was $<0.5 \mathrm{ng} / \mathrm{mL}$, thereby suggesting the possibility of bacterial infection to be less likely. On the 6th admission day, a CT scan of the neck detected crownshaped calcifications surrounding the odontoid process (Fig. 1a) and the linear calcification of the transverse ligament of the atlas (Fig. 1b). Although clinically asymptomatic without a previous history of arthritis, the patient's X- 

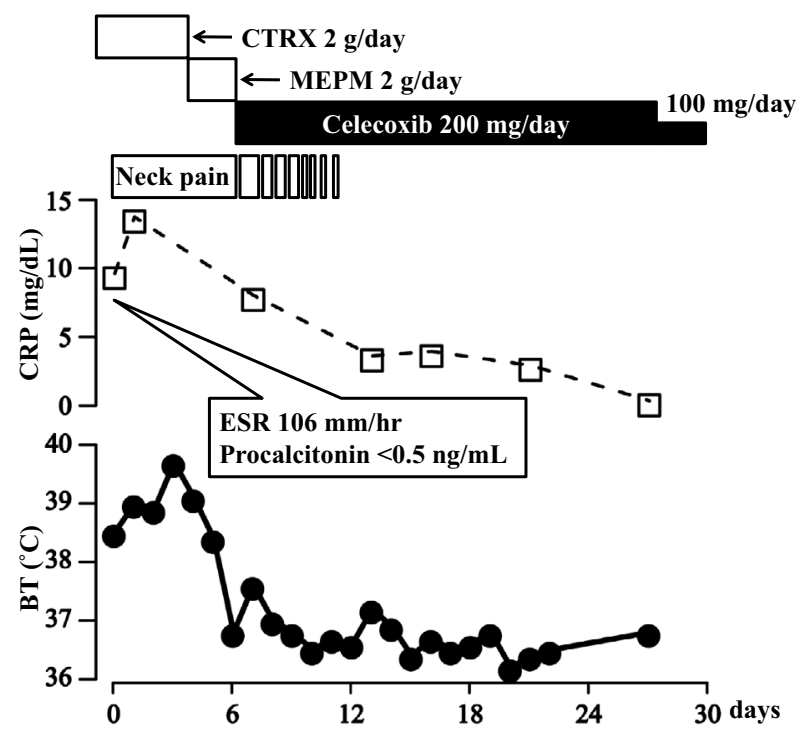

Figure 2. Clinical course. The administration of intravenous antibiotic agents (ceftriaxone sodium hydrate and meropenem hydrate) was ineffective. After NSAIDs therapy (200 $\mathrm{mg}$ of celecoxib per day) was started, the patient's high fever went down and the posterior neck pain improved immediately. The patient's neck pain disappeared completely 5 days after initiating NSAIDs therapy. Additionally, the CRP level decreased to within normal range 28 days later, and thereafter celecoxib was reduced to $100 \mathrm{mg}$ daily. BT: body temperature, CRP: Creactive protein, CTRX: ceftriaxone sodium hydrate, ESR: erythrocyte sedimentation rate, MEPM: meropenem hydrate

ray of the wrist, elbow, shoulder (Fig. 1c) and knee (Fig. 1d) joints revealed calcium deposits, suggesting underlying crystalline deposition disease. A diagnosis of CDS was made according to her clinical and radiological findings and treatment with nonsteroidal anti-inflammatory drugs (NSAIDs) was started (celecoxib, $200 \mathrm{mg}$ per day) on the same day. Her posterior neck pain and fever drastically improved within 5 days after initiating NSAIDs treatment and the serum inflammatory reaction level decreased to within normal range 28 days later (Fig. 2).

\section{Discussion}

CDS was initially reported in 1985 as feverish cervical pain caused by crystalline deposits in soft tissue around the odontoid process with inflammatory reactions (1). The components of the crystalline deposits is typically calcium pyrophosphate dehydrate (CPPD) or hydroxyapatite (HA) (3). The contribution of fibroblasts or chondroid cells in the ligaments around the odontoid process was proposed as a mechanism of calcium deposition $(4,5)$ and it is plausible to say that an increase of calcium deposits around the odontoid process leads to the expansion of soft tissue and mechanical damage to the anatomical structures, resulting in inflammatory reaction (6). However, the exact pathophysiology of CDS remains unclear.

Because its principal symptoms are acute posterior neck pain and fever, CDS may be misdiagnosed as meningitis $(2,6)$. In addition to the neck pain and fever, if morning stiffness in the shoulder girdle or jaw claudication is present, CDS may also mimic polymyalgia rheumatica (PMR) or giant cell arteritis (GCA) $(7,8)$. Despite the fact that our patient presented with high fever and posterior neck pain with inflammatory reactions, we did not perform a lumbar puncture because she lacked the symptoms of consciousness disturbance, neck stiffness or Kernig's sign. Furthermore, her procalcitonin level was less than $0.5 \mathrm{ng} / \mathrm{mL}$. Takahashi et al. reported a case of CDS mimicking aseptic meningitis whose measurement of procalcitonin was useful in excluding the infectious etiology for the differential diagnosis of CDS (2). However, the currently available data on the procalcitonin level in CDS patients is quite limited, thus further accumulation of case reports for this relatively rare clinical entity is necessary to avoid the performance of any unnecessary invasive diagnostic procedures.

A previous medical history of crystalline deposition disease in other joints is crucial information for the clinician to suspect $\operatorname{CDS}(1,2,5-8)$. Although the present case had never experienced acute crystalline arthritis before admission, an X-ray of her wrist, elbow, shoulder and knee joints revealed significant calcium deposits. Ferrone et al. reported that CPPD disease is not necessarily symptomatic (9). According to our patient's previous medical history, she had never experienced acute crystalline arthritis. Though X-rays revealed calcium deposits in several major joints, the deformity or OA-like change was not severe, suggesting the lack of chronic inflammation or previous episodes of repeated acute crystalline arthritis. Unfortunately, 67-Ga scintigraphy was not performed in this patient, therefore the presence of another focus for the inflammatory reaction could not be completely ruled out. Considering the physical findings and the results from the enhanced CT scan, UCG, blood culture, serological testing, the procalcitonin level and the clinical course, we believe this patient's inflammatory reaction was therefore attributable solely to CDS. The present case demonstrates that CDS may be an initial manifestation of an acute episode of crystalline deposition disease, and even though our patient was clinically asymptomatic with no apparent previous history of acute crystalline arthritis, an X-ray survey of the major joints was helpful for supporting the diagnosis of CDS.

A cervical CT scan is the golden standard for the diagnosis of CDS owing to its capability to detect calcium deposits around the odontoid process $(1,2,5-8)$. Moreover, a CT scan is helpful for ruling out other causes of neck pain, such as fractures and primary or metastatic tumors. Salaffi et al. reported that calcification around the odontoid process was observed in 25 out of 49 CPPD patients (51\%) and neck symptoms were present in 9 (18.4\%) cases (10). However, whether asymptomatic calcifications ultimately lead to the onset of CDS is uncertain. It is reasonable to assume that the growth of crystalline deposits around the odontoid process results in evoking the inflammatory reaction and causes 
symptoms such as neck pain, restriction of neck movement, and fever. Recently, CDS has been suggested to be more common than previously thought. Thus, clinicians should keep in mind the possibility of future incidences of CDS in patients with calcium deposits around the odontoid process even if they are asymptomatic at the time of examination.

The appropriate treatment for CDS remains debatable; however, NSAIDs are conventionally used for CDS $(3,5,8)$. In most of the previously reported cases, oral NSAIDs therapy alone was sufficient for the improvement of symptoms within several days. In some refractory cases, corticosteroids were additionally administered (6). In our case, the patient's fever promptly went down one day after celecoxib administration and her neck pain and restriction of cervical movement disappeared five days later. The patient's serum CRP level normalized within one month after treatment. Because CDS mainly occurs in elderly people, steroid therapy should be prescribed with careful consideration to avoid fatal side effects. Additionally, the long-term usage of anti-inflammatory drugs is unnecessary for the treatment of CDS (11). Clinicians should therefore be cautious about the choice and period of anti-inflammatory therapy for the treatment of CDS.

Although rare, CDS should be considered especially in elderly patients with fever and posterior neck pain when making a differential diagnosis of infectious meningitis to avoid any unnecessary invasive procedures or ineffective antibiotic treatment. CDS may occur as an initial presentation of crystalline deposition disease. Measuring the procalcitonin levels and the detection of calcium deposits in major joints by X-rays, even if the patient is asymptomatic and has no previous history of pseudogout attack, may be helpful for supporting the diagnosis of CDS.

The authors state that they have no Conflict of Interest (COI).

\section{References}

1. Bouvet JP, le Parc JM, Michalski B, Benlahrache C, Auquier L. Acute neck pain due to calcifications surrounding the odontoid process: the crowned dens syndrome. Arthritis Rheum 28: 1417$1420,1985$.

2. Takahashi T, Minakata Y, Tamura M, Takasu T, Murakami M. A rare case of crowned dens syndrome mimicking aseptic meningitis. Case Rep Neurol 5: 40-46, 2013.

3. Lee GS, Kim RS, Park HK, Chang JC. Crowned dens syndrome: a case report and review of the literature. Korean J Spine 11: 1517, 2014.

4. Doherty M, Dieppe P. Crystal deposition disease in the elderly. Clin Rheum Dis 12: 97-116, 1986.

5. Constantin A, Bouteiller G. Acute neck pain and fever as the first manifestation of chondrocalcinosis with calcification of the transverse ligament of the atlas. Five case-reports with a literature review. Rev Rhum Engl Ed 65: 583-585, 1998.

6. Sato Y, Yasuda T, Konno S, Kuwayama A, Komatsu K. Pseudogout showing meningoencephalitic symptoms: crowned dens syndrome. Intern Med 43: 865-868, 2004.

7. Pego-Reigosa JM, Rodriguez-Rodriguez M, Hurtado-Hernandez Z, et al. Calcium pyrophosphate deposition disease mimicking polymyalgia rheumatica: a prospective followup study of predictive factors for this condition in patients presenting with polymyalgia symptoms. Arthritis Rheum 53: 931-938, 2005.

8. Aouba A, Vuillemin-Bodaghi V, Mutschler C, De Bandt M. Crowned dens syndrome misdiagnosed as polymyalgia rheumatica, giant cell arteritis, meningitis or spondylitis: an analysis of eight cases. Rheumatology (Oxford) 43: 1508-1512, 2004.

9. Ferrone C, Andracco R, Cimmino MA. Calcium pyrophosphate deposition disease: clinical manifestations. Reumatismo 63: 246252, 2012.

10. Salaffi F, Carotti M, Guglielmi G, Passarini G, Grassi W. The crowned dens syndrome as a cause of neck pain: clinical and computed tomography study in patients with calcium pyrophosphate dihydrate deposition disease. Clin Exp Rheumatol 26: 10401046, 2008.

11. Matsumura M, Hara S. Crowned dens syndrome. N Engl J Med 367: e34, 2012.

(C) 2015 The Japanese Society of Internal Medicine http://www.naika.or.jp/imonline/index.html 